\title{
Local Density of States in Superconductor - Ferromagnetic Hybrid Systems
}

\author{
Rosario Fazio and Carlo Lucheroni \\ Istituto di Fisica, Università di Catania, viale A. Doria 6, I-95129 Catania, Italy \\ Istituto Nazionale per la Fisica della Materia (INFM), Unità di Catania, Italy
}

\begin{abstract}
In this Letter we discuss various properties of the local density of states (DOS) for a superconductor-ferromagnet hybrid system. The DOS is modified at small energies on both sides of the interface. Due to the interplay of superconductivity and ferromagnetism, the local DOS depends on the spin direction. The spin polarization effects extend over a long distance from the interface both in the superconductor and in the ferromagnet. If the ferromagnet is of finite lenght, the DOS shows a (spin dependent) gap.
\end{abstract}

PACS numbers: 74.80.Fp,72.50.Bg, 74.50.+r

Typeset using REVTEX 
When a normal metal is in contact with a superconductor, pairing correlations appear on the normal side. The proximity effect is intimately related to the microscopic mechanism which governs the transport through $\mathrm{SN}$ interfaces. An incoming electron from $\mathrm{N}$ is reflected at the interface as a quasi-hole, and a Cooper pair is injected into the superconductor. Physical consequences of the proximity effect have been investigated since the early days of superconductivity 1 . Nevertheless, thanks to the development of nanofabrication technology, there has been a tremendous interest in exploring mesoscopic effects in hybrid Superconductor-Normal metal (SN) hybrid structurest. This activity lead to the discovery of new phenomena as the magnetoresistance oscillations in $\mathrm{SN}$ system ${ }^{3}$ and the reentrant effect国目.

Due to the proximity effect, there is a modification of the local density of states (DOS)

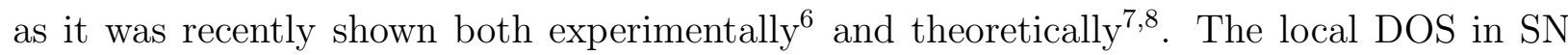
systems depends on the position relative to the interface. In the normal metal the DOS is suppressed at low energies whereas in the superconductor new states appear below the BCS gap. Properties of level statistics in hybrid system have been studied in Refs. 9:10.

The proximity effect is very sensitive to the presence of a strong electron-electron interaction, as in the case of nanostructures11, and to pair-breaking effects. In this Letter we focus on this last aspect by considering the case in which the metal is an itinerant ferromagnet. Properties of ferromagnetic-superconductor (FS) hybrid structures have been intensively investigated in the past 2 . It has been shown, for instance, that in FS bilayers and multilayers 13 the critical temperature and critical current oscillate as a function of the exchange field and thinkness of the ferromagnet. Both effects are related to the center of mass momentum aquired by the Cooper pair due to the Zeeman splitting. Further consequences of this phase shift can be observed in mesoscopic structures where the Andreev reflected hole retains its phase coherence with the incident electron 5 . 16 . Recent experiments suggest long-range coherence effects also in FS structures 17 and show a pronounced reentrant effect in transport for a ferromagnetic wire in contact with a superconductort 8 . Furthermore the presence of the exchange field leads to additional structure in the subgap current-voltage 
characteristic 19 .

An experiment which measures the local DOS in an FS system similarly to that of Ref. 6 seems to be feasable and it may lead, in our opinion, to further understanding of proximity in ferromagnetic materials. In this Letter we therefore study this problem by solving selfconsistently the Usadel equations for an FS interface. We will show that the combined effects of superconductivity (which introduces an energy dependence in the DOS) and ferromagnetism (which results in a finite momentum for the Cooper pairs entering the ferromagnet) leads to a rich structure in the DOS. The effects discussed in this Letter occur when the exchange energy is of the order of the superconducting gap. There is also a magnetic proximity effect which, for instance, induces a spin dependent DOS also on the superconducting side. Zeeman splitting of the DOS of magnetic materials and superconductors has been intesively investigated 20. Most of the attention was devoted to tunnel junctions. In this Letter we concentrate on the opposite limit, a highly transparent FS bilayer shown schematically in the inset of Fig. 11.

In the absence of an applied magnetic field the physical quantities depend only on the relative distance $x$ from the interface. Superconductor and ferromagnet have finite length, $L_{S}$ and $L_{F}$ respectively. The ferromagnet is described by the Stoner model which consists of an effective one-electron Hamiltonian in an exchange field. For simplicity we assume that the only effect of electron-electron interaction is to create the exchange field and therefore we ignore any residual interaction which can induce a finite superconducting gap on the $\mathrm{F}$ side.

In the dirty limit, the quasiclassical retarded Green's function $\hat{g}(x, E)$ satisfies the Usadel equation21

$$
D \partial_{x} \hat{g} \cdot \partial_{x} \hat{g}+i\left(E+\mu_{B} H(x)\right)\left[\hat{\tau}_{z}, \hat{g}\right]+[\hat{\Delta}(x), \hat{g}]=0
$$

with the constraints $\hat{g} \hat{g}=1$ and $\operatorname{Tr} \hat{g}=0$. The hat refers to the the Nambu notation $\left(\hat{\tau}_{z}\right.$ and $\hat{\tau}_{y}$ are the Pauli pseudospin matrices). In eq.(1) $D$ is the diffusion constant, $\mu_{B}$ is the Bohr magneton, $H(x)$ is a magnetic exchange field (we assume that all the material parameters 
are identical on both sides of the interface). The gap matrix $\hat{\Delta}(x)=\Delta(x) \hat{\tau}_{y}$ is determined self-consistently ( $\Delta(x)$ can be chosen to be real) by means of

$$
\Delta(x)=\lambda(x) \int_{0}^{\omega_{D}} d E \operatorname{Im}\left[g_{12}(x, E, H)+g_{12}(x, E,-H)\right]
$$

where $\lambda(x)=\lambda \Theta(-x)\left(\lambda=\operatorname{arcosh}\left(\omega_{D} / \Delta_{B C S}\right)\right.$ is the BCS coupling constant, $\Theta(x)$ is a step function), $\Delta_{B C S}$ the BCS gap and $\omega_{D}$ is the Debye cutoff frequency. The exchange field $H(x)=H \Theta(x)$ is not determined self-consistently, hence we do not consider the possible modification of the domain structure in the ferromagnet due to the presence of superconductivity 22. In the problem under consideration, there are two important energy scales, the Zeeman energy $\mu_{B} H$ and the BCS gap $\Delta_{B C S}$. Associated with them there are two typical length scales $\xi_{F}=\sqrt{D / 2 \mu_{B} H}$ (the coherence length in the ferromagnet) and $\xi_{S}=\sqrt{D / 2 \Delta_{B C S}}$ (the superconducting correlation length). Since we study the case of perfect transmission at the FS interface the Green's functions and their derivatives are continuous at $x=0$.

The local DOS $N_{\uparrow(\downarrow)}$ for spins parallel $(\uparrow)$ and antiparallel $(\downarrow)$ to the exchange field $H$ are

$$
N_{\uparrow(\downarrow)}(x, E)= \pm N_{0} \operatorname{Re} g_{11,(22)}(x, \pm E, H)
$$

where $N_{0}$ is the DOS (per spin) of a normal metal at the Fermi surface. We solve the problem at zero temperature. Then, as long as the temperature is kept well below the critical temperature of the superconductor, all our results (possibly smeared by inelastic scattering) are valid.

The scattering at the FS interface causes pairbreaking2 2 . The gap $\Delta$ is suppressed close to the interface more strongly than in to the SN case. In the ferromagnet the pair amplitude $F(x)=\left\langle\psi_{\uparrow}(x) \psi_{\downarrow}(x)\right\rangle$ will be non-vanishing. In Fig. 1 the self-consistent gap is calculated for different values of the dimensionless exchange field $h=\mu_{B} H / \Delta_{B C S}$. By increasing $h$, the value of $\Delta$ close to the interface is suppressed and it almost vanishes for the largest exchange field which we consider. The scale at which the superconducting gap reaches the bulk value is $\xi_{S}$ (independent of the exchange field). It is possible that for stronger exchange fields (we 
are currently investigating this issue), $\Delta(x)$ may show a nontrivial behaviour close to the interface. Indeed this would not a new situation. A superconductor in a uniform exchange field may have different forms of pairing. In the Larkin-Ovchinnikov-Fulde-Ferrell state24, for instance, the gap is modulated with a period proportional to the exchange field.

For a Cooper pair entering the ferromagnet, the electron and the hole states are shifted by the Zeeman energy. As a consequence, the pair amplitude (i.e. the Cooper pair density) will show rapid oscillations on the scale of $\xi_{F}$ 14. In Fig.2 we show the results of our self-consistent calculation of the pair amplitude. On incresing the exchange field, the pair amplitude drops faster to zero and the period of the oscillation becomes shorter. Only the first oscillation is clearly seen while the others are strongly damped due to pairbreaking effects.

We now turn to a detailed discussion of the local DOS. The most important findings of Ref. 8 can be summarized as follows. i) The DOS in the normal and in the superconducting side of the system aquires a nontrivial dependence on the energy: the superconductor has a finite DOS below the gap while the normal metal has strong suppression DOS at the Fermi energy. ii) If the normal metal has a finite length $L$ there is a minigap in the excitation spectrum which scales as $\sim L^{-2}$. The value of the minigap does not depend of the distance from the interface. iii) The distance at which the anomaly in the DOS vanishes is energy dependent and it goes as $\sim \sqrt{D / 2 E}$.

All these effects are seen also in FS hybrid systems, with quantitative differences though. Here we concentrate on those properties of the DOS which depend on the presence of the intinerant ferromagnetism on the normal side. In particular we discuss the dependence of the DOS on the exchange field and the spin polarization effects. The DOS in the ferromagnet is shown in Fig. 3. Its behaviour for spin parallel and antiparallel to the exchange field is very different. Whereas for parallel spin the anomaly is strongly suppressed by the field, it is shifted to an energy of the order of $\mu_{B} H$ for antiparallel one. The anomaly is enhanced compared to the normal system $(H=0)$ (similarly to what observed in spin polarized tunneling experiments2). In Fig. 3 we chose $x=0.1 \xi_{S}$. We stress, however, that the anomalous behaviour of the DOS is long-range and persists over many correlation lengths. 
The anomaly is strongest when $\mu_{B} H \sim \Delta_{B C S}$, by further increasing $H$ it progressively shrinks and the DOS approaches a constant. Even if pairing correlations die out on scales of the order of $\xi_{F}$, the anomalies in DOS survive over a much longer length scale. The different behaviour of spin up and spin down electrons on the F side (the spin polarization effect) is shown in Fig. 团 where $\delta N=N_{\downarrow}(x, E)-N_{\uparrow}(x, E)$ is analyzed as a function of energy and position. The spin polarization of the DOS is entirely due to the proximity effect. Due to proximity the DOS aquires an energy dependence also on the normal side of the interface. The Zeeman splitting, due to the exchange fiels on the F side, leads to a different behaviour for the two spin directions.

There is also a magnetic proximity in the superconductor. A finite exchange field is induced close to the interface, up to distances of the order of the superconducting correlation length 3 . Here we emphasize another aspect of this proximity which occurs in the superconductor (see Fig. 5). Both in the F and $\mathrm{S}$ sides, spin polarization effects are most pronounced at energies of the order of the Zeeman splitting, and extend over many coherence lengths far from the interface.

The last issue we address is the behaviour of the DOS when the ferromagnet has a finite length. Similarly to the SN system, a gap $E_{G}$ opens at the Fermi energy. On the superconducting side, the gap shrinks approaching the interface from the bulk. On the normal side, the gap does not depend on the distance from the interface. The gap location and width, however, do depend on the spin direction and on the value of the exchange field. We only concentrate on the gap which opens at the Fermi energy. For parallel spin $E_{G}$ is suppressed to zero when the (pair-breaking) exchange field is switched on (Fig. 6a) whereas for antiparallel spin it has a non-monotonous behaviour (Fig. [6b). The gap first increases by increasing the exchange field up to $h \sim 0.5$, then, for higher values of the exchange field it is rapidly closed. For larger fields the position of the gap is roughly centered around $\mu_{B} H$ for spin down electrons. We postpone a more detailed description of the DOS of FS bilayers and multilayers (including the important role of spin-orbit scattering) to a forthcoming publication 25 
In this Letter we studied the local DOS for a FS hybrid system. Most of the effects discussed here are pronounced if the exchange field is of the order of the superconducting gap $\Delta_{B C S}$ like in $\mathrm{YCO}_{2-x} \mathrm{Al}_{x}$ (which has a ferromagnetic transition around $10 \mathrm{~K}$ ). A system closely related is a planar thin-film SN in a parallel magnetic field. Besides the fact that a Zeeman splitting is present in the superconductor, most of the results of this remain valid and can be checked experimentally.

\section{ACKNOWLEDGMENTS}

We thank F. Beltram, W. Belzig, C. Bruder, G. Falci, A. Filip, G. Giaquinta, M. Giroud, F.W.J. Hekking, B. Pannetier and J. Siewert for many useful discussions. The support of the PAIS-EISS of INFM is gratefully acknowledged. 


\section{REFERENCES}

${ }^{1}$ G. Deutscher and P.G. de Gennes, in Superconductivity R.D. Parks Ed. (M Dekker, NY, 1969).

${ }^{2}$ C.W.J. Beenakker in Mesoscopic Quantum Physics, edited by E. Akkermans, G. Montambaux, and J.-L Pichard (North Holland, Amsterdam) 1995; R. Raimondi and C.J. Lambert, J. Phys. C 10, 901 (1998).

${ }^{3}$ V.T. Petrashov, V.N. Antonov, P. Delsing, and, T. Claeson, Phys. Rev. Lett. 70, 347 (1993).

${ }^{4}$ P. Charlat, H. Courtois, Ph. Gandit, D. Mailly, and B. Pannetier, Phys. Rev. Lett. 77, $4950(1996)$.

${ }^{5}$ T.H. Stoof and Yu.V. Nazarov, Phys. Rev. Lett. 76, 2981 (1996).

${ }^{6}$ S. Guéron, H. Pothier, N. O. Birge, D. Esteve, and M. Devoret, Phys. Rev. Lett. 77, 3025 (1996).

${ }^{7}$ A. A. Golubov and M. Yu. Kupriyanov, J. Low Temp. Phys. 70, 83 (1988).

${ }^{8}$ W. Belzig, C. Bruder, and G. Schön, Phys. Rev. B 54, 9443 (1996).

${ }^{9}$ K.M. Frahm, P.W. Brouwer, J.A. Melsen, and C.W.J. Beenakker, Phys. Rev. Lett. 77, $823(1996)$.

${ }^{10}$ A. Altland, B. D. Simons, D. Taras-Semchuk, JETP Lett. 67, 1 (1998).

${ }^{11}$ R. Fazio and G. Schön in Mesoscopic Electron Transport, NATO ASI series E, Vol, 345, p. 407, Kluver (1997).

${ }^{12}$ L.N. Bulaevskii, A.I. Buzdin, M.L. Kulíc, and S.V. Panyukov Adv. Phys. 34, 175 (1985).

${ }^{13}$ Z. Radovic, M. Ledvij, L. Dobrosavljević-Grujic̀, A.I. Buzdin, and J.R. Clem, Phys. Rev. B 54, 9443 (1996). 
${ }^{14}$ E.A. Demler, G.B. Arnold, and M.R. Beasley, Phys. Rev. B 55, 15174 (1997).

${ }^{15}$ M.D.M. de Jong and C.W.J. Beenakker, Phys. Rev. Lett. 74, 1657 (1995).

${ }^{16}$ S.K. Upadhyay, A. Palanisami, R.N. Louie, and R.A. Buhrman, Phys. Rev. Lett. 81, 3247 (1998).

${ }^{17}$ V.T. Petrashov, JETP Lett. 59, 551 (1994); M.D. Lawrence and N. Giordano, J. Phys. C.8, 563 (1996).

${ }^{18}$ M. Giroud, H. Courtois, K. Hasselbach, D. Mailly and B. Pannetier, Phys. Rev. B 58, R11872 (1998).

${ }^{19}$ M. Leadbeater, C. J. Lambert, R. Raimondi, A. F. Volkov, cond-mat/9811117.

${ }^{20}$ R. Meservey and P.M. Tedrow, Phys. Rep. 238, 173 (1994).

${ }^{21}$ A. Schmid in Nonequilibrium Superconductivity, Phonons, and Kapitza Boundaries, NATO ASI Series B 65, ed. K.E. Gray, (Plenum, New York 1981).

${ }^{22}$ A.I. Buzdin and L.N. Bulaevskii, Sov. Phys. JETP 67, 576 (1988).

${ }^{23}$ T. Tokuyasu, J.A. Sauls, and D. Rainer, Phys. Rev. B 38, 8823 (1988).

${ }^{24}$ A.I. Larkin and Yu. N. Ovchinnikov, Sov. Phys. JETP 20, 762 (1965); P. Fulde and R.A. Ferrell, Phys. Rev. A 135, 550 (1964).

${ }^{25}$ R. Fazio and C. Lucheroni, in preparation. 


\section{FIGURES}

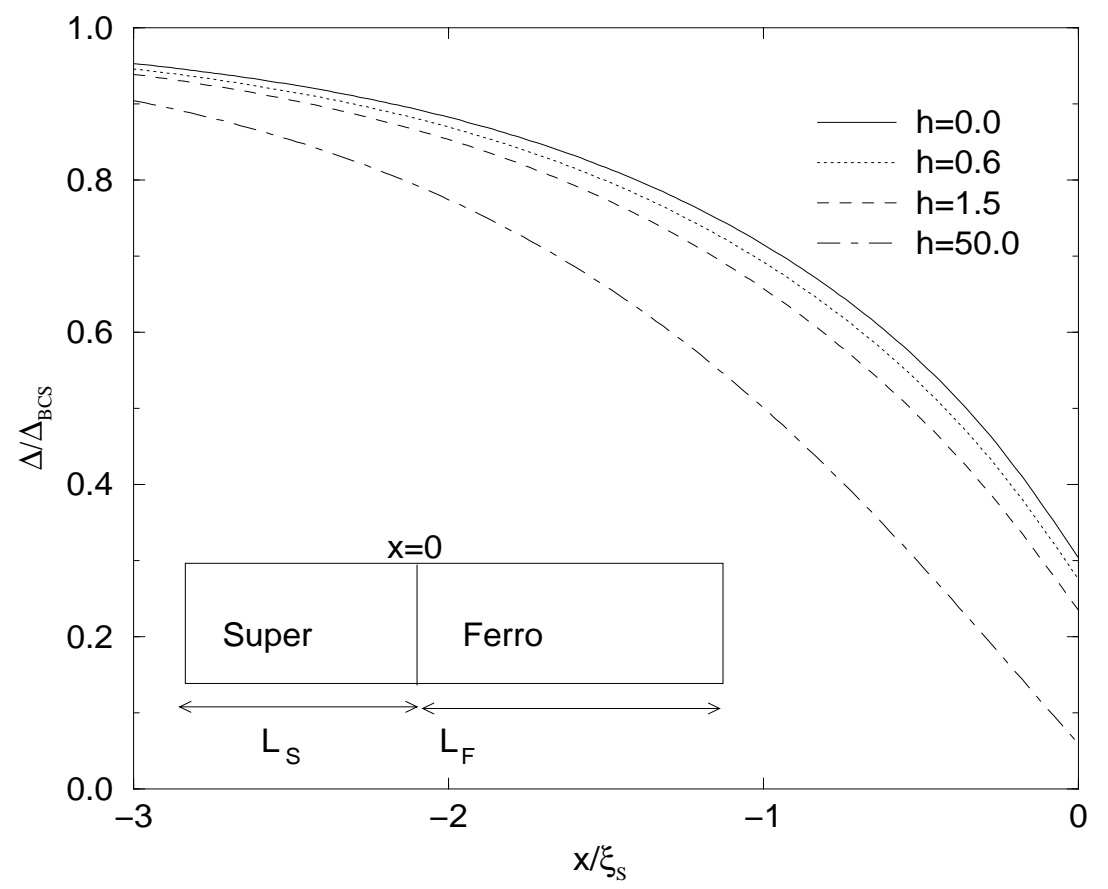

FIG. 1. The self-consistent gap is shown as a function of the distance from the interface for various values of $h$. The system has dimensions $L_{F}=L_{S}=10 \xi_{S}$. Inset: the hybrid FS system considered in this work. On the l.h.s. the superconductor, on the r.h.s. the ferromagnet.

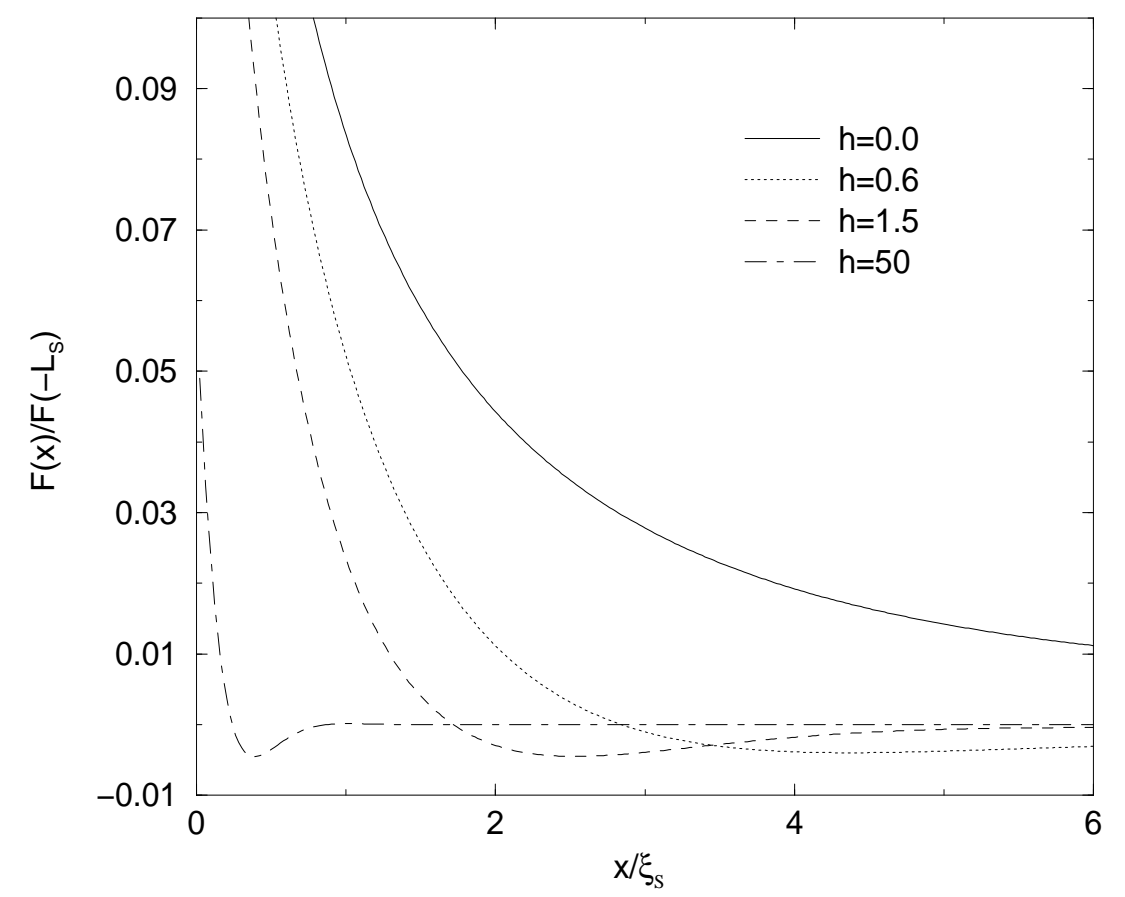

FIG. 2. Pair amplitude on the ferromagnetic side for several values of the exchange field $\left(L_{S}=10\right.$ and $\left.L_{F}=10\right)$. 


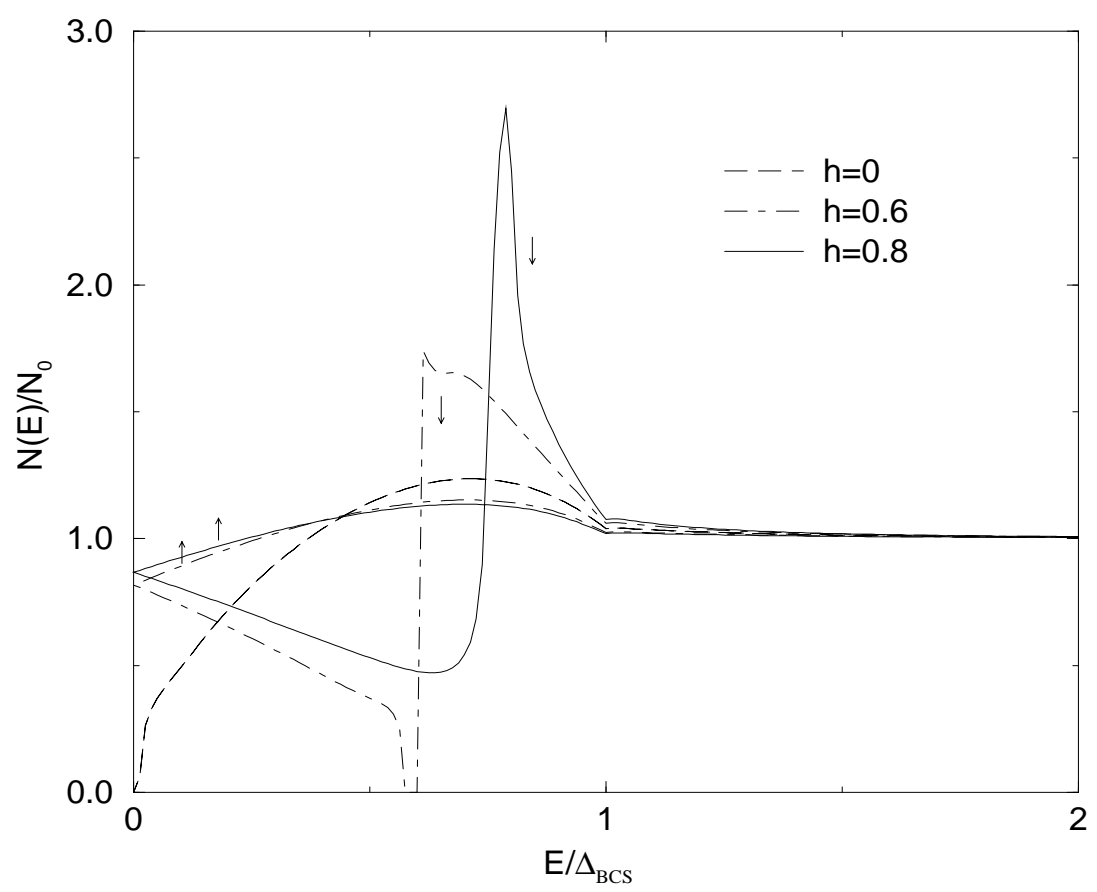

FIG. 3. DOS for the system with $L_{S}=10, L_{F}=10$ on the ferromagnetic side, close to the interface $\left(x=0.1 \xi_{S}\right)$, for different values of the exchange fields, $(\uparrow / \downarrow)$ refer to parallel and antiparallel spin respectively. Compared to the normal metal $(h=0)$, the anomalies in the DOS are enhanced by the ferromagnetic correlations.

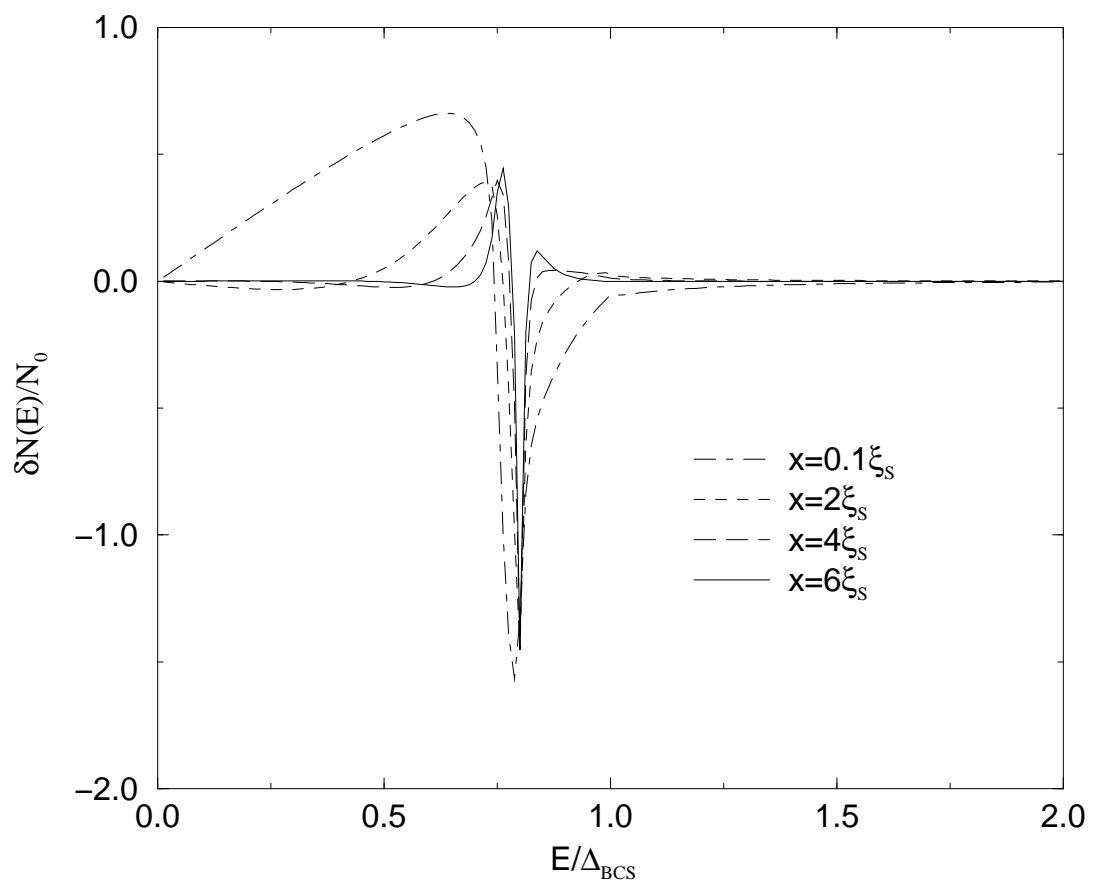

FIG. 4. The different in the DOS for opposite spin directions $\delta N$, at different distances from the interface on the $\mathrm{F}$ side $\left(L_{S}=10, L_{F}=10, h=0.8\right)$. 


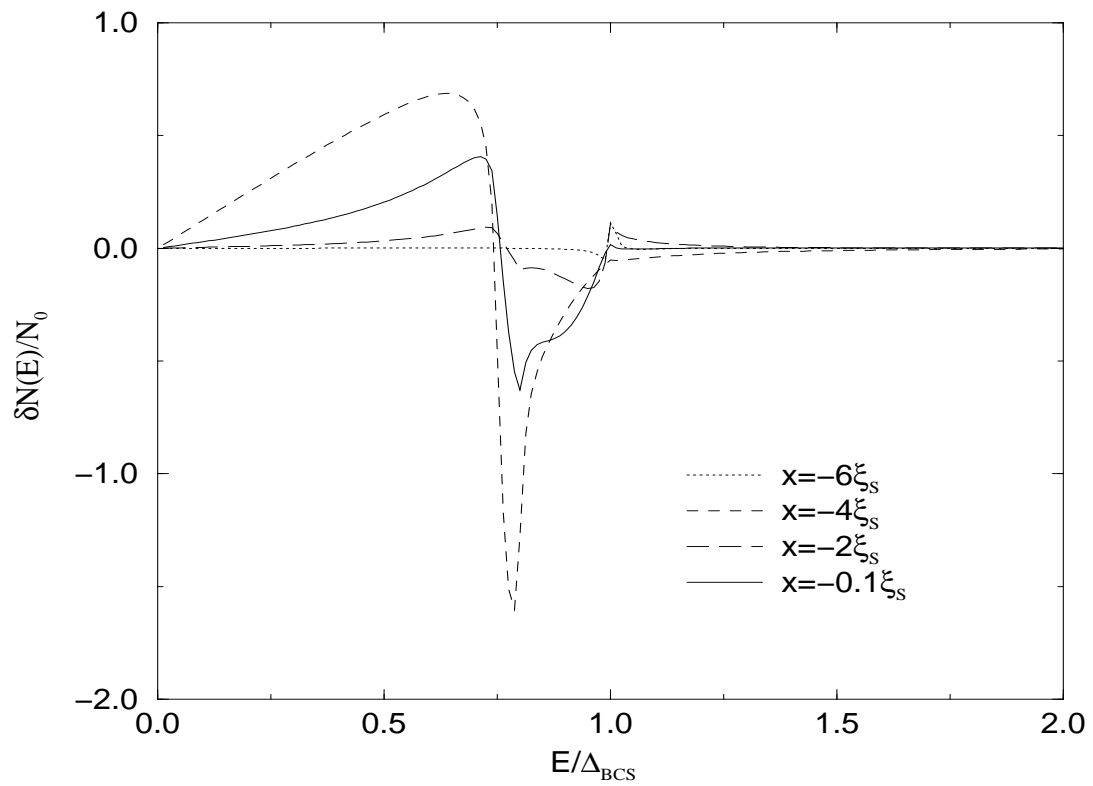

FIG. 5. The same as in Fig. 1 on the $\mathrm{S}$ side $\left(L_{S}=10, L_{F}=10\right), h=0.8$.

(a) $\uparrow$

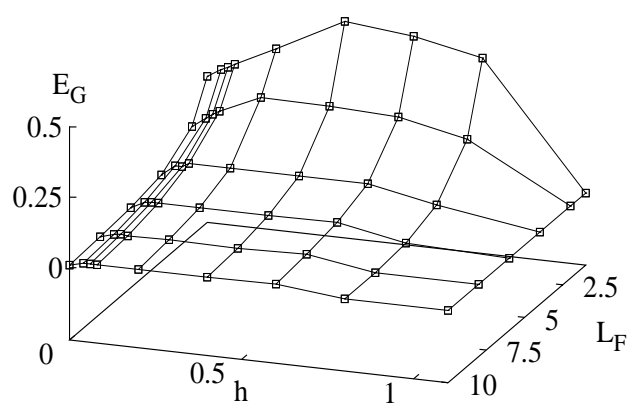

(b) $\downarrow$

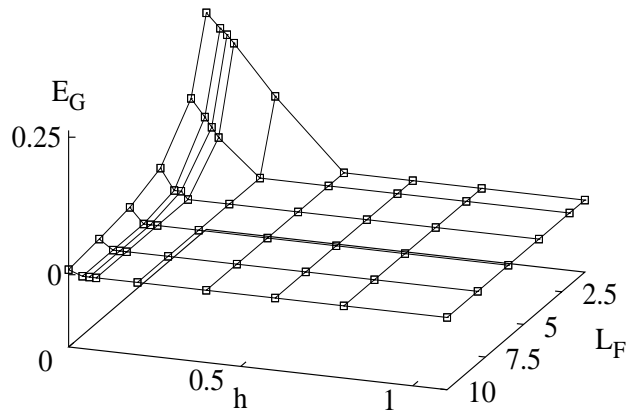

FIG. 6. Behaviour of the gap as a function of length of the normal metal and the exchange field $\left(L_{S}=10\right) .(\uparrow)$ parallel spins, $(\downarrow)$ antiparallel spins. 